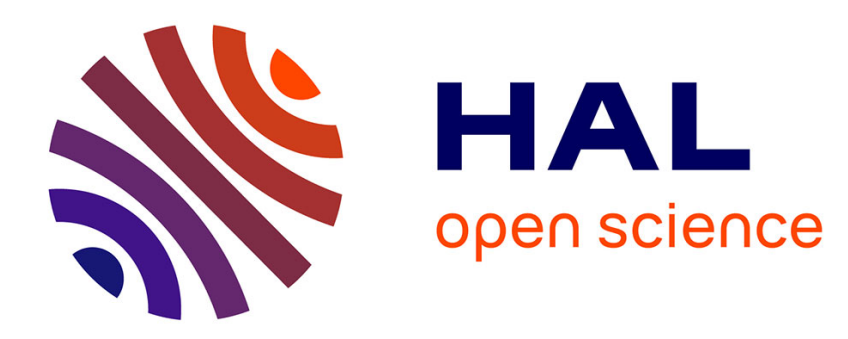

\title{
Renormalization along the chemical sequence of a single polymer chain
}

\author{
M. Gabay, T. Garel
}

\section{To cite this version:}

M. Gabay, T. Garel. Renormalization along the chemical sequence of a single polymer chain. Journal de Physique Lettres, 1978, 39 (9), pp.123-125. 10.1051/jphyslet:01978003909012300 . jpa-00231458

\section{HAL Id: jpa-00231458 https://hal.science/jpa-00231458}

Submitted on 1 Jan 1978

HAL is a multi-disciplinary open access archive for the deposit and dissemination of scientific research documents, whether they are published or not. The documents may come from teaching and research institutions in France or abroad, or from public or private research centers.
L'archive ouverte pluridisciplinaire HAL, est destinée au dépôt et à la diffusion de documents scientifiques de niveau recherche, publiés ou non, émanant des établissements d'enseignement et de recherche français ou étrangers, des laboratoires publics ou privés. 


\title{
LE JOURNAL DE PHYSIQUE-LETTRES
}

\author{
Classification \\ Physics Abstracts \\ $61.40-64.70$

\section{RENORMALIZATION ALONG THE CHEMICAL SEQUENCE OF A SINGLE POLYMER CHAIN}

\author{
M. GABAY and T. GAREL $\left({ }^{*}\right)$ \\ Laboratoire de Physique des Solides (**), Université Paris-Sud, Centre d’Orsay, 91405 Orsay, France
}

(Reçu le 12 janvier 1978, accepté le 8 mars 1978)

\begin{abstract}
Résumé. - Nous avons calculé, à l'ordre $\varepsilon(\varepsilon=4-d)$, l'exposant $v$ pour un polymère isolé avec volume exclu, à l'aide d'une transformation de renormalisation le long de la chaîne. Nous pouvons ainsi suivre le passage entre les comportements Gaussien et non-Gaussien. Les résultats sont en accord avec ceux de la méthode $" n=0 »$.
\end{abstract}

\begin{abstract}
We have derived, to order $\varepsilon(\varepsilon=4-d)$, the exponent $v$ for a single polymer chain with excluded volume, by means of a renormalization transformation along the chain. As a consequence, we can follow the crossover from Gaussian to non-Gaussian behaviour. These results agree with those obtained by the $" n=0$ method".
\end{abstract}

1. Introduction. - It is well-known that the net effect of Van der Waals interactions in a polymer chain may be an effect of repulsion, leading to an expansion of the chain [1]. The two parameters of interest in this problem can be chosen as :

(i) $\left\langle L^{2}\right\rangle$ the mean square end-to-end length of the chain.

(ii) $X$ the strength of the repulsive interaction between links (monomers).

Roughly speaking, two types of theoretical approach have been used to treat the excluded volume problem. On the one hand, various perturbation expansions have been performed based on three-dimensional (3d) models [1]. On the other, this problem has been related to Wilson's theory of critical phenomena [2]. In this note, we wish to present a renormalization group (RG) transformation along the chain [3]. This is a perturbation expansion in $d$ dimensions, combined with Kadanoff's block concept [4, 5]. In section 2 , we recall briefly some basic results of the two previous methods. The RG transformation and its consequences for the Gaussian to non-Gaussian crossover are studied in section 3.

2. Summary of previous results. $-2.13 \mathrm{~d}$ PERTURBATION EXPANSIONS. - A simple model which describes the effect of $X$ on $\left\langle L^{2}\right\rangle$ is that of Fixman [6]. He considers a chain of $g$ links $(g \gg 1)$. The ith

$\left(^{*}\right)$ Also at : SPSRM, C.E.N. Saclay, B.P. $n^{\circ} 2,91190$ Gif sur Yvette, France.

$\left({ }^{* *}\right)$ Laboratoire associé au C.N.R.S. link is represented by a vector $r_{i}$, of mean value $\left\langle r_{i}^{2}\right\rangle=b^{2}$. When one assumes random flight statistics for the $r_{i}$ and a contact $\left({ }^{1}\right)$ repulsive interaction of magnitude $X$, one obtains to first order :

where

$$
\left\langle L^{2}\right\rangle=g b^{2}+C_{1} X
$$

$$
C_{1}=\frac{4}{3} b^{2}\left(\frac{3}{2 \pi b^{2}}\right)^{3 / 2} g^{3 / 2}
$$

To carry on this expansion, an important remark should be made : although the interaction $X$ between links is of the short range type in space, it is of the long range type along the chain. Therefore, the magnitude of the effective repulsive interaction is smaller than $X$. Indeed, Kurata et al. [7] have calculated the second virial coefficient $A_{2}$, which, to order $X^{2}$, reads :

$$
A_{2} \sim X\left(1-2.865\left(\frac{3}{2 \pi b^{2}}\right)^{3 / 2} g^{1 / 2} X\right) .
$$

A self-consistent approach to the excluded volume expansions should therefore make use of this last equation.

2.2 Analogy With CRITICAL PHENOMENA. - De Gennes [2] has shown that the excluded volume problem can be mapped onto the critical behaviour of an

( $\left.{ }^{1}\right)$ The interaction energy is : $k_{\mathrm{B}} T X \sum \Sigma_{i>j} \delta\left(\mathbf{L}_{i j}\right)$, where $\mathbf{L}_{i j}$ connects the $i$ th and $j$ th links. $X$ has therefore the dimension of a volume. 
$n$-vector spin model [4], in the limit $n \rightarrow 0$. Thus, the exponent $v$ defined by $\left\langle L^{2}\right\rangle \sim g^{2 v}(g \gg 1)$ is given to first order in $\varepsilon(\varepsilon=4-d)$ by

$$
2 v=1+\frac{\varepsilon}{8} \text {. }
$$

Above the critical dimensionality $d_{\mathrm{c}}=4$, random walk (Gaussian) behaviour is expected.

3. Renormalization transformation along the chain. - 3.1 Definition. - Let us consider a chain of $N$ links $(N \rightarrow \infty)$ in a $d$ dimensional space. Following Kadanoff [4], we divide it into blocks of $g$ links, and consider these blocks as the new links. We then iterate the procedure. After the pth step, the blocks will be characterized, to lowest order, by a length

$$
\left\langle L_{p}^{2}\right\rangle=g\left\langle L_{p-1}^{2}\right\rangle
$$

and a repulsive interaction $X_{p}=g^{2} X_{p-1}$.

A dimensionless quantity [8] is :

$$
\frac{X_{p}}{\left(\left\langle L_{p}^{2}\right\rangle\right)^{d / 2}}=g^{\left(\frac{4-d}{2}\right)} \frac{X_{p-1}}{\left(\left\langle L_{p-1}^{2}\right\rangle\right)^{d / 2}} .
$$

This suggests that the effect of repulsion is irrelevant for $d>4$.

3. 2 APPROXimATE RECURSION RELATIONS.- When one constructs blocks of $g$ links, one has to consider three types of interactions (Fig. 1) : intrablock interactions (Fig. 1a), nearest-neighbour interblock interactions (Fig. 1b), and other interblock interactions (Fig. 1c). Recalling Kadanoff's method, we are led, in the first place, to neglect interactions of the type (1b). Transposing the results of section 2.1 to a $d$-dimensional space, we obtain :

$$
\begin{aligned}
\left\langle L_{p+1}^{2}\right\rangle & =g\left\langle L_{p}^{2}\right\rangle\left[1+\left(\frac{d}{2 \pi}\right)^{d / 2} \times\right. \\
& \left.\times \frac{4}{(4-d)(6-d)} g^{\left(\frac{4-d}{2}\right)} \frac{X_{p}}{\left(\left\langle L_{p}^{2}\right\rangle\right)^{d / 2}}\right] \\
X_{p+1}= & g^{2} X_{p}\left[1-\left(\frac{d}{2 \pi}\right)^{d / 2} \times\right. \\
\quad \times & \left.\left.\frac{32}{(2-d)(4-d)(6-d)}-1\right\} g^{\left(\frac{4-d}{2}\right)} \frac{X_{p}}{\left(\left\langle L_{p}^{2}\right\rangle\right)^{d / 2}}\right] .
\end{aligned}
$$

As shown below, these equations are valid to first order in $\varepsilon(\varepsilon=4-d)$. This is why we have set $X_{p}$ instead of $X_{p+1}$ in (1) and $\left\langle L_{p}^{2}\right\rangle$ instead of $\left\langle L_{p+1}^{2}\right\rangle$ in (2).

Let us define

$$
\alpha_{p}=\left(\frac{d}{2 \pi}\right)^{d / 2} g^{\left(\frac{4-d}{2}\right)} \frac{X_{p}}{\left(\left\langle L_{p}^{2}\right\rangle\right)^{d / 2}} .
$$

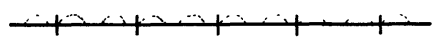

(1a)
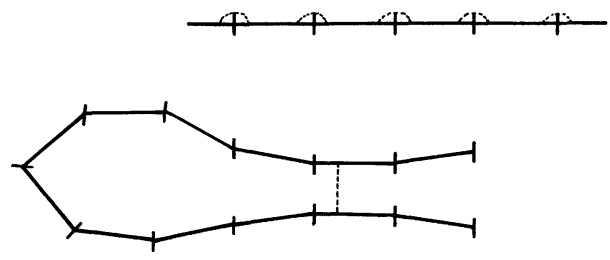

(1c)

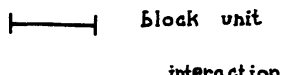

FIG. 1. - The three types of interactions involved in the block procedure.

Then, from (1) and (2) :

$$
\begin{aligned}
& \alpha_{p+1}=g^{\left(\frac{4-d}{2}\right)} \alpha_{p}\left[1-\left\{\frac{2 d}{(4-d)(6-d)}+\right.\right. \\
& \left.\left.+\frac{32}{(2-d)(4-d)(6-d)}\left(\frac{2^{\left(\frac{8-d}{2}\right)}-2}{8-d}-1\right)\right\} \alpha_{p}\right] .
\end{aligned}
$$

When $\varepsilon \ll 1$, one has :

$$
\begin{aligned}
\left\langle L_{p+1}^{2}\right\rangle & =g\left\langle L_{p}^{2}\right\rangle\left(1+\frac{2}{\varepsilon} \alpha_{p}\right) \\
\alpha_{p+1} & =g^{\varepsilon / 2} \alpha_{p}\left(1-\frac{8}{\varepsilon} \alpha_{p}\right) .
\end{aligned}
$$

As suggested by de Gennes [3], a correspondence can be drawn between Wilson's RG approach and the one we have used here. Therefore, we look for the non-trivial fixed point of (4) :

$$
\alpha_{\infty}=\frac{\varepsilon^{2}}{16} \operatorname{Ln} g \text {. }
$$

The eigenvalue associated with this (stable) fixed point is $-\varepsilon / 2$. Inserting (5) into (3) yields :

$$
\operatorname{Lim}_{p \rightarrow \infty} \frac{\left\langle L_{p+1}^{2}\right\rangle}{\left\langle L_{p}^{2}\right\rangle}=g^{1+\varepsilon / 8}
$$

i.e.

$$
2 v=1+\frac{\varepsilon}{8} .
$$

At this point, an important remark should be made. The coefficient of $\alpha_{p}$ in equations (3) and (4) behaves, quite unusually, like $1 / \varepsilon$. Therefore, it is not obvious that first order in $\alpha_{p}$ means first order in $\varepsilon$. This is why we have evaluated the coefficient of $\alpha_{p}^{2}$ in (3) and (4); we find an expression of the type :

$$
\frac{A}{\varepsilon^{2}}+\frac{B}{\varepsilon}+C
$$

where $A ; B ; C$ are numerical constants. Together with (5), this indicates that the $\alpha_{p}^{2}$ term does not 
generate $O(\varepsilon)$ terms in (3) and (4). We are fairly confident that this remains true for higher powers of $\alpha_{p}$.

3.3 Crossover Regime. - Through numerical iteration, recursion relations (3) and (4) allow us to follow the crossover between Gaussian and nonGaussian behaviour. Universality predicts that

$$
\left(\left\langle L^{2}\right\rangle\right)^{1 / 2}=\left(\left\langle L_{\mathrm{G}}^{2}\right\rangle\right)^{1 / 2} f\left(\alpha_{0} N^{\varepsilon / 2}\right)
$$

where $\left\langle L_{\mathbf{G}}^{2}\right\rangle \sim N$ denotes the length of the chain in the Gaussian case; $f(x)$ is the crossover function, and $\alpha_{0}$ is the initial value of the dimensionless parameter $\alpha_{p}$. In figure 2 , we have plotted $\left(\left\langle L^{2}\right\rangle\left\langle\left\langle L_{\mathrm{G}}^{2}\right\rangle\right)^{1 / 2}\right.$ as a function of $x=\alpha_{0} N^{\varepsilon / 2}$ for $\varepsilon=1$. The results fit with the following law [10] :

For

$$
x<2 \times 10^{-2} \quad f(x)=(1+\lambda x)^{\beta}
$$

where $\lambda \sim 27.5$ and $\beta \sim 0.123$.

For $x$ large, $\beta$ remains approximately constant but $\lambda$ is changed $(\lambda \sim 18$ for $x \sim 3)$, because of higher-order corrections.

These results agree with those of Stephen [9], who using the $n=0$ analogy, has calculated $f(x)$ to first order in $\varepsilon$, and predicts $\beta=\frac{1}{8}$.

4. Conclusion. - We have presented a derivation, to first order in $\varepsilon(\varepsilon=4-d)$, of the exponent $v$

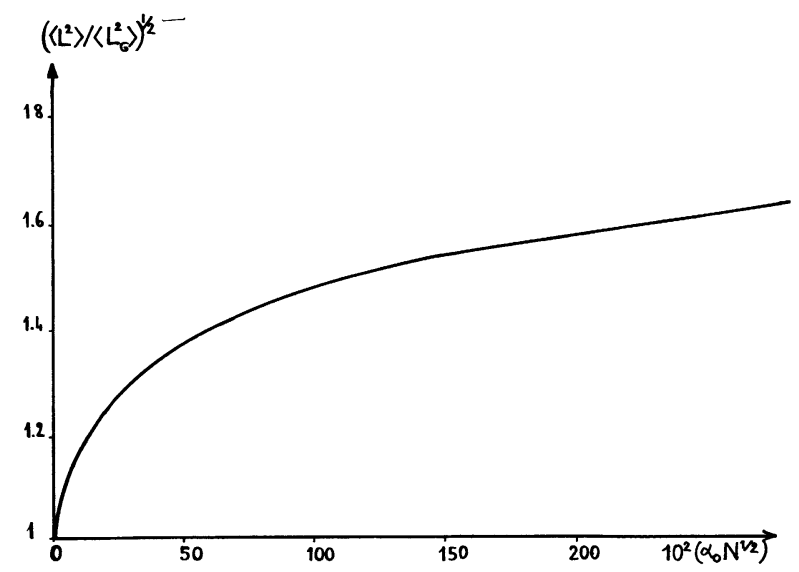

Fig. 2. - Plot of $\left(\left\langle L^{2}\right\rangle\left\langle\left\langle L_{\mathrm{G}}^{2}\right\rangle\right)^{1 / 2}\right.$ versus $10^{2}\left(\alpha_{0} N^{1 / 2}\right)$ as deduced from the recursion relations.

and of the crossover function $f(x)$ for the excluded volume problem, without using the $n=0$ analogy. The method, which makes use of a renormalization transformation along the chain, may be extended to other related problems ( $\theta$ point, solutions, ...). Further work in this direction is in progress.

Acknowledgments. - We thank Dr. J. des Cloizeaux for many relevant remarks, Pr. P. G. de Gennes for fruitful comments on the validity of the approximations, and Dr. P. Pfeuty for interesting discussions.

\section{References}

[1] A summary of the various methods is given in :

FloRY, P. J., Principles of Polymer Chemistry (Cornell University Press N.Y.) 1966.

[2] DE Gennes, P. G., Phys. Lett. 38A (1972) 339.

[3] This idea was given during lectures by Pr. de Gennes and Dr. des Cloizeaux, see also

DE Gennes, P. G., Riv. Nuovo Cimento 7 (1977) 363.

[4] Kadanoff, L. P., Physics 2 (1966) 263.

[5] Wilson, K. G., Kogut, J., Phys. Rep. 12C (1974) 77.

[6] Fixman, M., J. Chem. Phys. 23 (1955) 1656.
[7] Kurata, M., Fukatsu, M., Sotobayashi, H., Yamakawa, H., J. Chem. Phys. 41 (1964) 139.

[8] We wish to thank Dr. des Cloizeaux for discussion on this point.

[9] Stephen, M. J., Phys. Lett. 53A (1975) 363.

[10] Note that we have assumed the constants in eqs. (1) and (2) to take their $d=4$ values to obtain equations (3) and (4). This explains why the value of $\lambda$ does not agree with the first-order calculation of section 2.1. For a different matching procedure, see :

Burch, D. J., Moore, M. A., J. Phys. A 9 (1976) 435. 